\title{
Flow Equations and Normal Ordering
}

\author{
Elmar Körding $\ddagger$ and Franz Wegner \\ Institut für Theoretische Physik, Universität Heidelberg, Philosophenweg 19, D-69120 \\ Heidelberg
}

\begin{abstract}
In this paper we consider flow-equations where we allow a normal ordering which is adjusted to the one-particle energy of the Hamiltonian. We show that this flow converges nearly always to the stable phase. Starting out from the symmetric Hamiltonian and symmetry-broken normal ordering nearly always yields symmetry breaking below the critical temperature.
\end{abstract}

PACS numbers: 64.60, 05.30, 71.10

\section{Introduction}

In applying flow equations to a Hamiltonian [1] one typically starts out from a Hamiltonian which does not show an explicit symmetry breaking even if the symmetry of the system will be broken below some temperature. As in the calculations on the Hubbard model [2, 3, 4, 5] one can direct the flow to a form in which molecular-field approximation becomes exact. This effective Hamiltonian is still not symmetry broken. Only the molecular-field formalism breaks the symmetry below the critical temperature $T_{c}$.

In the fermionic renormalization group flow [6, 7, 8, 9, 10, the vertex functions will at least within weak-coupling approximations diverge at some length scale below $T_{c}$, so that the approximations become unreliable and one has to resort to other methods in this regime. Thus it is desirable to have a way to introduce symmetry breaking from the beginning. Recently Salmhofer, Honerkamp, Metzner, and Lauscher [12] have added a symmetry breaking field to the Hamiltonian and showed that this additional field leads into the symmetry broken phase.

For the Hamiltonian flow it is not necessary to add a symmetry breaking term to the Hamiltonian. Instead it is sufficient to choose a normal-ordering which is symmetry broken (compare [11]). One can show that the system will nearly always converge to the stable state, that is in case of symmetry breaking (that is below the critical temperature) it runs to a symmetry broken state, whereas if the symmetric state is stable (above $T_{c}$ ) then it will run to the symmetric state.

With this approach the normal ordered form of the Hamiltonian shows an explicit dependence on the normal ordering. We adjust continuously the normal ordering to $\ddagger$ present address: School of Physics and Astronomy, University of Southampton, SO17 1BJ, UK 
the one-particle contribution of the Hamiltonian and we can show that the normal ordering has the interesting property to run in the direction of the stable state. This implies that starting out with a symmetry-broken normal ordering, then above the critical temperature it will converge to the symmetric state whereas below criticality it converges to a symmetry broken state.

We start out from the flow equation [1]

$$
\frac{\mathrm{d} H(l)}{\mathrm{d} l}=[\eta(l), H(l)]
$$

where $\eta$ is chosen so that the Hamiltonian approaches a diagonal or block-diagonal form. We write $H$ in the normal-ordered form

$$
H=: H_{G}:_{G}
$$

where $G$ defines the normal ordering

$$
G_{k j}=\left\langle a_{k} a_{j}\right\rangle_{0}
$$

Here we denote both creation and annihilation operators by $a$. Then the flow equation reads

$$
\frac{\mathrm{d} H_{G}}{\mathrm{~d} l}=[\eta, H]_{G}+\frac{\delta H_{G}}{\delta G} \frac{\partial G}{\partial l} .
$$

The second term indicates the change of $H_{G}$ due to the change of $G$. $G$ itself can be described as the expectation values of corresponding to a bilinear Hamiltonian $H^{0}$. Then the flow equation (41) is supplemented by a second equation for $H^{0}$. This equation describes the adaption of $H^{0}$ to the one-particle energy of $H$, and will be given later (46).

In the next section we consider the effect of normal ordering on operators. Then we give the general relation between a bilinear Hamiltonian and the expectation values in thermal equilibrium. Then we return to the flow equation and show that almost always the normal ordering will approach a stable state.

\section{Normal Ordering}

The idea behind normal ordering is to subtract expectation values

$$
G_{k j}=\left\langle a_{k} a_{j}\right\rangle
$$

from products of operator pairs $a_{k}, a_{j}$. More precisely one defines normal ordering which is indicated by two colons by

$$
\begin{array}{lll}
: 1: & =1 \\
: \alpha A(a)+\beta B(a): & =\alpha: A(a):+\beta: B(a): \\
a_{k}: A(a): & =: a_{k} A(a):+\sum_{j} G_{k j}: \frac{\partial}{\partial a_{j}} A(a):
\end{array}
$$


where $\alpha, \beta$ are c-numbers. These equations hold for bosons and fermions. In performing the derivatives for fermions one has to consider that the operators $a$ anticommute. Iteration of (8) yields

$$
a_{k_{1}} a_{k_{2}} \ldots a_{k_{m}}=:\left(a_{k_{1}}+\sum_{l_{1}} G_{k_{1} j_{1}} \frac{\partial}{\partial a_{j_{1}}}\right)\left(a_{k_{2}}+\sum_{j_{2}} G_{k_{2} j_{2}} \frac{\partial}{\partial a_{j_{2}}}\right) \ldots a_{k_{m}}:
$$

which can also be written

$$
a_{k_{1}} a_{k_{2}} \ldots a_{k_{m}}=: \exp \left(\sum_{k j} G_{k j} \frac{\partial^{2}}{\partial a_{j}^{\text {right }} \partial a_{k}^{\text {left }}}\right) a_{k_{1}} a_{k_{2}} \ldots a_{k_{m}}:
$$

This is Wick's first theorem [13. The superscripts left and right indicate that we always pick a pair of factors $a$ and perform the derivative $\frac{\partial}{\partial a_{k}}$ on the left factor and the derivative $\frac{\partial}{\partial a_{j}}$ on the right factor, so that the factor $G_{k j}$ depends on the sequence of the operators. The exponential appears in the equation for the following reason. If we perform the operation $G \frac{\partial^{2}}{\partial a \partial a}$ on $m$ pairs of factors $a$, then there are due to the permutation symmetry $m$ ! contributions. Therefore in order to obtain the contribution with factor one we have to divide the $m$-th power of $G \frac{\partial^{2}}{\partial a \partial a}$ by $m$ !, which yields the exponential. Note that for fermions the operators $a$ as well as the derivatives $\frac{\partial}{\partial a}$ anticommute.

Since in the following we will change the normal-ordering it is appropriate to indicate to which expectation values $G$ it is performed. Then we obtain quite general for operators $A$

$$
: A_{G}:_{G}=A, \quad A_{G}=\exp \left(\sum_{k j} G_{k j} \frac{\partial^{2}}{\partial a_{j}^{\text {right }} \partial a_{k}^{\text {left }}}\right) A(a)
$$

If we dissect $G_{k j}=Q_{k j}+C_{k j}$, where $C_{k j}=C_{j k}$ for bosons and $C_{k j}=-C_{j k}$ for fermions, then we have

$$
: A_{G}:_{G}=: A_{Q}:_{Q}, \quad A_{G}=\exp \left(\frac{1}{2} \sum_{k j} C_{k j} \frac{\partial^{2}}{\partial a_{j} \partial a_{k}}\right) A_{Q} .
$$

and we need no longer distinguish the sequence of the factors. Suppose we have

$$
H=v^{(0)}+\frac{1}{2 !} \sum_{k j} v_{k j}^{(1)} a_{k} a_{j}+\frac{1}{4 !} \sum_{k j m n} v_{k j m n}^{(2)} a_{k} a_{j} a_{m} a_{n}
$$

and $v_{k j}^{(1)}$ and $v_{k j m n}^{(2)}$ are completely symmetric for bosons and antisymmetric for fermions, resp., then the normal-ordered form reads

$$
\begin{aligned}
& H_{G}=v_{G}^{(0)}+\frac{1}{2 !} v_{G, k j}^{(1)} a_{k} a_{j}+\frac{1}{4 !} v_{G, k j m n}^{(2)} a_{k} a_{j} a_{m} a_{n}, \\
& v_{G}^{(0)}=v^{(0)}+\frac{1}{2} \sum_{k j} v_{k j}^{(1)} G_{k j}+\frac{1}{8} \sum_{k j m n} v_{k j m n}^{(2)} G_{k j} G_{m n}, \\
& v_{G, k j}^{(1)}=v_{k j}^{(1)}+\frac{1}{2} \sum_{m n} v_{k j m n}^{(2)} G_{m n}, \\
& v_{G, k j m n}^{(2)}=v_{k j m n}^{(2)} .
\end{aligned}
$$


Expressing $A$ and $B$ by $A_{G}$ and $B_{G}$ by means of eq. (10) and then transforming back to the normal-ordering we obtain for the product of two operators

$$
: A_{G}(a):_{G}: B_{G}(a):_{G}=: \exp \left(\sum_{k j} G_{k j} \frac{\partial^{2}}{\partial b_{j} \partial a_{k}}\right) A_{G}(a) B_{G}(b):\left.\right|_{G, b=a} \text {. }
$$

\section{Expectation values for $H^{0}$}

In the following we will consider only fermions. A conventional way to introduce normal ordering is to use a Hamiltonian

$$
H^{0}=\frac{1}{2} \tilde{\epsilon}_{k j} a_{k^{*}} a_{j}
$$

where we use the notation

$$
a_{k}^{\dagger}=a_{k^{*}}, \quad a_{k}=a_{k^{*}}^{\dagger} .
$$

Considering $a$ a column vector and $a^{\dagger}$ a row vector we may write

$$
\begin{gathered}
\tilde{\epsilon}_{k j} a_{k^{*}} a_{j}=\left(\begin{array}{cc}
a^{\dagger} & a^{T}
\end{array}\right) \tilde{\epsilon}\left(\begin{array}{c}
a \\
a^{*}
\end{array}\right)=\left(\begin{array}{ll}
a^{\dagger} & a^{T}
\end{array}\right)\left(\begin{array}{cc}
A & B \\
B^{\dagger} & -A^{T}
\end{array}\right)\left(\begin{array}{c}
a \\
a^{*}
\end{array}\right) \\
A^{\dagger}=A, \quad B^{T}=-B .
\end{gathered}
$$

Thus $\tilde{\epsilon}$ has the properties

$$
\tilde{\epsilon}^{\dagger}=\tilde{\epsilon}, \quad \tau \tilde{\epsilon} \tau=-\tilde{\epsilon}^{T}, \quad \tau=\left(\begin{array}{cc}
0 & 1 \\
1 & 0
\end{array}\right)
$$

$\tilde{\epsilon}$ can be diagonalized with diagonal matrix elements $\epsilon_{k}$ and $-\epsilon_{k}$. (This diagonalization is performed by a canonical transformation which is isomorphic to a real orthogonal transformation. This can be easily seen if one introduces the hermitean linear combinations $q_{k}=\frac{a_{k}^{\dagger}+a_{k}}{\sqrt{2}}$ and $p_{k}=\frac{\mathrm{i}\left(a_{k}^{\dagger}-a_{k}\right)}{\sqrt{2}}$.)

In thermal equilibrium one obtains for diagonal $\tilde{\epsilon}$, i.e. $\tilde{\epsilon}_{k j}=\delta_{k j} \epsilon_{k}$

$$
\left\langle a_{k}^{\dagger} a_{k}\right\rangle=\frac{1}{\mathrm{e}^{\beta \epsilon_{k}}+1}, \quad\left\langle a_{k} a_{k}^{\dagger}\right\rangle=\frac{1}{\mathrm{e}^{-\beta \epsilon_{k}}+1}
$$

so that even when $\tilde{\epsilon}$ is not diagonal

$$
\tilde{G}=\frac{1}{\mathrm{e}^{\beta \tilde{\epsilon}}+1}=\frac{1}{2}-\frac{1}{2} \tanh \left(\frac{\beta \tilde{\epsilon}}{2}\right)
$$

holds with $\tilde{G}_{k j}=G_{k^{*} j}$. A variation of $\tilde{\epsilon}$ yields

$$
\delta \tilde{G}=-\frac{1}{\mathrm{e}^{\beta \tilde{\epsilon}}+1} \delta \mathrm{e}^{\beta \tilde{\epsilon}} \frac{1}{\mathrm{e}^{\beta \tilde{\epsilon}}+1}=-\frac{1}{\mathrm{e}^{\beta \tilde{\epsilon}}+1} \int_{0}^{\beta} \mathrm{d} \tau \mathrm{e}^{\tau \tilde{\epsilon}} \delta \tilde{\epsilon} \mathrm{e}^{(\beta-\tau) \tilde{\epsilon}} \frac{1}{\mathrm{e}^{\beta \tilde{\epsilon}}+1} .
$$

Thus we may write

$$
\delta \tilde{G}_{k j}=-\Gamma_{k j, p q} \delta \tilde{\epsilon}_{p q}
$$

with

$$
\Gamma_{k j, p q}=\int_{0}^{\beta} \mathrm{d} \tau\left(\frac{1}{\mathrm{e}^{\beta \tilde{\epsilon}}+1} \mathrm{e}^{\tau \tilde{\epsilon}}\right)_{k p}\left(\mathrm{e}^{(\beta-\tau) \tilde{\epsilon}} \frac{1}{\mathrm{e}^{\beta \tilde{\epsilon}}+1}\right)_{q j} .
$$




\section{Free Energy and Stability}

It is well known, that for a given Hamiltonian $H$ and temperature $T$ the free energy assumes its minimum for the corresponding statistical operator $\rho=\mathrm{e}^{-\beta H} / Z$. Thus one often determines approximately the free energy for the statistical operator $\rho^{0}=$ $\mathrm{e}^{-\beta H^{0}} / Z^{0}$ with $H^{0}$ bilinear in the operators $a$ and determines $\tilde{\epsilon}$ so that the corresponding free energy $F^{0}$ becomes minimal. One obtains

$$
F^{0}=E-T S, \quad E=v_{G}^{(0)}, \quad S=-\frac{k_{\mathrm{B}}}{2} \operatorname{tr}(\tilde{G} \ln \tilde{G}+(1-\tilde{G}) \ln (1-\tilde{G}))
$$

We use that $\langle A(a)\rangle^{0}=\left.A_{G}\right|_{a=0}$. We will vary this expression. By means of

$$
\begin{aligned}
A_{G+\delta G} & =A_{G}+\frac{1}{2} \sum_{k j} \delta G_{k j} \frac{\partial^{2}}{\partial a_{j} \partial a_{k}} A_{G} \\
& +\frac{1}{8} \sum_{k j m n} \delta G_{k j} \delta G_{m n} \frac{\partial^{4}}{\partial a_{j} \partial a_{k} \partial a_{n} \partial a_{m}} A_{G}+O\left(\delta G^{3}\right)
\end{aligned}
$$

we obtain

$$
E=v_{G}^{(0)}+\frac{1}{2} v_{G, k^{*} j}^{(1)} \delta \tilde{G}_{k j}+\frac{1}{8} v_{G, k^{*} j m^{*} n}^{(2)} \delta \tilde{G}_{k j} \delta \tilde{G}_{m n}+\ldots
$$

A variation of $S$ yields in first order in $\delta \tilde{G}$

$$
\delta S=-\frac{k_{\mathrm{B}}}{2} \ln \left(\frac{\tilde{G}}{1-\tilde{G}}\right)_{j k} \delta \tilde{G}_{k j}
$$

Therefore we obtain in first order in $\delta \tilde{G}$

$$
\begin{aligned}
\delta F^{0} & =\frac{1}{2}\left(v_{G, k^{*} j}^{(1)}+k_{\mathrm{B}} T \ln \left(\frac{\tilde{G}}{1-\tilde{G}}\right)_{j k}\right) \delta \tilde{G}_{k j} \\
& =\frac{1}{2}\left(v_{G, k^{*} j}^{(1)}-\tilde{\epsilon}_{k j}\right) \delta \tilde{G}_{k j} .
\end{aligned}
$$

In order that $F^{0}$ is an extremum one has to choose $\tilde{\epsilon}_{k j}=v_{G, k^{*} j}^{(1)}$, that is the one-particle contribution of our Hamiltonian $H_{G}$ has to agree with $H^{0}$. In second order in $\delta \tilde{G}$ we obtain

$$
\delta F^{0}=\frac{1}{8} v_{G, k^{*} j m^{*} n}^{(2)} \delta \tilde{G}_{k j} \delta \tilde{G}_{m n}-\frac{1}{4} \delta \tilde{\epsilon}_{k j} \delta \tilde{G}_{k j},
$$

where $\delta \tilde{\epsilon}$ is given in terms of $\delta \tilde{G}$ from (26)

$$
\delta \tilde{\epsilon}_{k j}=-\left(\Gamma^{-1}\right)_{k j, m n} \delta \tilde{G}_{m n}
$$

which yields

$$
\delta F^{0}=\frac{1}{4}\left(\frac{1}{2} v_{G, k^{*} j m^{*} n}^{(2)}+\left(\Gamma^{-1}\right)_{k j, m n}\right) \delta \tilde{G}_{k j} \delta \tilde{G}_{m n} .
$$

Only if this expression is positive definite then it corresponds to a stable solution. We note that $\Gamma$ is positive definite and therefore $\Gamma^{-1}$ exists and is positive definite, too. This can be seen if we switch to the basis in which $\tilde{\epsilon}$ is diagonal, $\tilde{\epsilon}_{k j}=\delta_{k j} \epsilon_{k}$. Then

$$
\begin{aligned}
\Gamma_{k j, p q} & =\delta_{k p} \delta_{j q} \Gamma_{k j}^{\mathrm{d}}, \\
\Gamma_{k j}^{\mathrm{d}} & =\frac{\mathrm{e}^{\beta \epsilon_{k}}-\mathrm{e}^{\beta \epsilon_{j}}}{\left(\mathrm{e}^{\beta \epsilon_{k}}+1\right)\left(\epsilon_{k}-\epsilon_{j}\right)\left(\mathrm{e}^{\beta \epsilon_{j}}+1\right)}>0 .
\end{aligned}
$$


Thus $\Gamma$ is diagonal with positive matrix elements along the diagonal.

These expressions are closely connected to the response function in random phase approximation. Suppose one adds a perturbation $\delta v^{(1 \mathrm{ext})}$ to the Hamiltonian $H$ which produces a change $\delta \tilde{G}^{\text {ext }}$ in the expectation values $\left\langle a_{k} a_{j}\right\rangle$ then we have from eqs. (3336)

$$
\frac{1}{2}\left(\delta v_{G, k^{*} j}^{(1, \text { ext })}+\left(\frac{1}{2} v_{G, k^{*} j m^{*} n}^{(2)}+\left(\Gamma^{-1}\right)_{k j, m n}\right) \delta \tilde{G}_{m n}\right) \delta \tilde{G}_{k j}^{\text {ext }}=0 .
$$

If we now relate $\delta \tilde{G}^{\text {ext }}$ to a perturbation $\delta v^{(1, \text { eff })}$ which would have the same effect without the interaction $v^{(2)}$ according to eq. (26)

$$
\delta \tilde{G}_{m n}^{\mathrm{eff}}=-\Gamma_{m n, p q} \delta v_{p q}^{(1, \mathrm{eff})}
$$

then we obtain

$$
\delta v_{G, k^{*} j}^{(1, \mathrm{ext})}=\left(\frac{1}{2} v_{G, k^{*} j m^{*} n}^{(2)} \Gamma_{m n, p q}+\delta_{k p} \delta_{j q}\right) \delta v_{p^{*} q}^{(1, \mathrm{eff})}
$$

This factor $\frac{1}{2} v^{(2)} \Gamma+1$ enters for example in the Lindhard expression for the static dielectric constant. One has only to introduce $v^{(2)} \propto e^{2} / q^{2}$ and

$$
\Gamma_{k j}^{\mathrm{d}}=\frac{f\left(\epsilon_{j}\right)-f\left(\epsilon_{k}\right)}{\epsilon_{k}-\epsilon_{j}}
$$

with the Fermi function $f(\epsilon)$. The dielectric constant has to be positive for a stable system.

\section{Flow Equations}

Now we have to introduce our flow equations. We have two contributions to the change of $H_{G}$, one from the generator of the flow,

$$
[\eta, H]_{G}=g^{(0)}+\frac{1}{2} g_{k j}^{(1)} a_{k} a_{j}+\ldots
$$

and one from the change of the normal-ordering which yields

$$
\frac{1}{2} \frac{\partial G_{k j}}{\partial l} \frac{\partial^{2}}{\partial a_{j} \partial a_{k}} H=-\frac{1}{2} \Gamma_{k j, m n} \frac{\partial \tilde{\epsilon}_{m n}}{\partial l}\left(v_{k^{*} j}^{(1)}+\frac{1}{2} v_{k^{*} j p^{*} q}^{(2)} a_{p^{*}} a_{q}+\ldots\right)
$$

Thus we obtain the change of $v^{(1)}$

$$
\frac{\partial v_{k^{*} j}^{(1)}}{\partial l}=g_{k^{*} j}^{(1)}-\frac{1}{2} \Gamma_{p q, m n} v_{p^{*} q k^{*} j}^{(2)} \frac{\partial \tilde{\epsilon}_{m n}}{\partial l}
$$

for the one-particle energy. On the other hand we wish to adapt $\tilde{\epsilon}$ so that it approaches $v^{(1)}$. Therefore we introduce a flow equation for $\tilde{\epsilon}$

$$
\frac{\partial \tilde{\epsilon}_{k j}}{\partial l}=\gamma\left(v_{k^{*} j}^{(1)}-\tilde{\epsilon}_{k j}\right)
$$

with some positive constant $\gamma$. Evidently for fixed $v^{(1)}$ the energy $\tilde{\epsilon}$ approaches exponentially $v^{(1)}$. From both equations we obtain

$$
\frac{\partial\left(v_{k^{*} j}^{(1)}-\tilde{\epsilon}_{k j}\right)}{\partial l}=g_{k^{*} j}^{(1)}-\gamma\left(\delta_{k m} \delta_{j n}+\frac{1}{2} \Gamma_{p q, m n} v_{p^{*} q k^{*} j}^{(2)}\right)\left(v_{m^{*} n}^{(1)}-\tilde{\epsilon}_{m n}\right)
$$


Thus as $g^{(1)}$ from the generator of the flow decreases also $v^{(1)}-\tilde{\epsilon}$ will decrease provided the kernel $1+\frac{1}{2} \Gamma v^{(2)}$ is positive definite. Otherwise the difference $v^{(1)}-\tilde{\epsilon}$ will nearly always increase exponentially. The condition that this kernel is positive definite is equivalent to the stability obtained from $F^{0}$ in eq. (36) above, since the kernel for $F^{0}$ differs only by the factor $\Gamma$, which itself is positive definite.

[1] F. Wegner, Annalen der Physik (Leipzig) 3 (1994) 77

[2] I. Grote, E. Körding, and F. Wegner, J. Low Temp. Phys. 126 (2002) 1385

[3] V. Hankevych, I. Grote, and F. Wegner, Phys. Rev. B66 (2002) 094516

[4] V. Hankevych and F. Wegner, Acta Phys. Pol. B 34 (2003) 497, Erratum 34 (2003) 1591

[5] V. Hankevych and F. Wegner, Eur. Phys. Journal B 31 (2003) 497

[6] R. Shankar, Physica A 177 (1991) 530

[7] R. Shankar, Rev. Mod. Phys. 66 (1994) 129-192

[8] D. Zanchi and H.J. Schulz, Phys. Rev. B61 (2000) 13609

[9] C.J. Halboth, W. Metzner, Phys. Rev. B61 (2000) 7364

[10] M. Salmhofer and C. Honerkamp, Prog. Theor. Phys. 105 (2001) 1

[11] E. Körding, diploma thesis Heidelberg 2001

[12] M. Salmhofer, C. Honerkamp, W. Metzner, O. Lauscher, Prog. Theor. Phys. 112 (2004) 943-970

[13] G.C. Wick, Phys. Rev. 80 (1950) 268 